\title{
Event Detection Using Unmanned Aerial Vehicles: Ordered versus Self-organized Search
}

\author{
Evşen Yanmaz \\ Institute of Networked and Embedded Systems, Mobile Systems Group, \\ University of Klagenfurt, Austria \\ evsen.yanmaz@uni-klu.ac.at
}

\begin{abstract}
Event coverage problem in wireless sensor networks has drawn the interest of several researchers. While most of the previous work has been on static or ground mobile sensor networks, airborne sensor networks have also found its way into several civil and military applications such as environmental monitoring or battlefield assistance. In this work, we study the mobility pattern of an Unmanned Aerial Vehicle (UAV) network and explore the benefits of ordered and self-organized random mobility in terms of event detection performance, when the event is stationary and event duration is finite. Specifically, we compare the performance of a UAV network flying in parallel formation to a simple, distributed, locally-interactive coverage-based mobility model as well as legacy mobility models such as random walk and random direction. We study the event detection probability of the UAV network with both perfect and imperfect sensing capabilities. Our results show that when the timing constraints are highly stringent or when the UAV sensors have a high miss probability, flying in formation cannot achieve a high detection probability and a self-organized distributed mobility model is more effective.
\end{abstract}

\section{Introduction}

Wireless sensor networks have found various application venues in environmental monitoring, health monitoring, target tracking in hostile situations, etc. [1]-[2]. Especially, in the case of monitoring physically inaccessible or dangerous areas for humans to enter, such as wildfire tracking, glacier or volcano monitoring, liveliness detection in emergencies or hazardous material tracking, use of wireless sensor networks is expected to increase tremendously. Due to the inaccessibility of the geographical areas in these applications, the sensor nodes either need to be dropped forming a random static network or mobile ground or airborne robots equipped with sensors are needed to be deployed. Moreover, if the event (target) to be detected by the sensor network is of time-critical nature, the coverage of the network should be sufficiently high to be able to respond to the detected event in a timely manner; such as wildfire monitoring or liveliness detection under rubble in case of an earthquake, where the emergency personnel work against the clock. In such cases, using a mobile sensor network would be highly beneficial both in terms of event detection and utilization of the available system resources [3].

In this paper, we study the event detection performance of an unmanned aerial vehicle (UAV) network for different mobility models. Specifically, we aim to determine 
when it would be beneficial for the UAV network to fly in a centralized, deterministic, parallel-formation. To this end, first, we derive the probability of detection of the UAV network flying in formation, when a finite-duration stationary event is assumed to occur at a random location in the geographical area to be monitored. We assume that sensing capabilities of the sensors on-board are imperfect (i.e., there is a non-zero probability that a UAV will miss an event in its sensing coverage). We compare the performance of parallel-formation with a distributed, coverage-based, cooperative mobility model that operates in a self-organizing manner and uses only local topology information to detect events without prior knowledge of the physical topology, by reducing the overlapping covered areas. While determining the mobility path, no assumption is made on the application the sensor network is deployed for. Numerical studies are conducted to test the performance of the parallel-formation and coverage-based mobility models as well as legacy mobility models such as random walk and random direction. It is shown that while a centralized, deterministic, parallel-formation mobility model might be easier to implement, it does not always provide acceptable performance in terms of event detection probability. More specifically, when the event needs to be detected within a strict time interval or when the sensing capabilities on the UAVs are highly imperfect (unreliable) a more intelligent, adaptive, and preferably self-organizing mobility model is required to achieve a high probability of event detection. In such cases, our results show that the simple, distributed, random mobility models investigated in this paper can overcome the limitations of the parallel-formation model.

The remainder of the paper is organized as follows. In Section 2 background on mobility models and coverage problem in wireless sensor networks is summarized. A brief event detection analysis for the parallel-formation is provided in Section 3 . Results are given in Section 4 and the paper is concluded in Section 5

\section{Background}

\subsection{Coverage in Wireless Sensor Networks}

Coverage problems in wireless sensor networks are of great importance and have been investigated by several researchers. In static wireless sensor networks, in general, coverage problem is treated as a node-activation and scheduling problem [7]-[9]. More specifically, algorithms are proposed to determine which sensor nodes should be active such that an optimization criterion is satisfied. The criterion can for instance be minimizing the coverage time, achieving a certain event detection probability, or covering each point in the area by at least $k$ sensors, etc. In addition, there are also studies that take into account not only the event (or network) coverage, but the connectivity of the wireless sensor network as well [7]. While deciding which sensor nodes should be active at a given point in time, coverage and connectivity requirements are met.

Recently, mobile sensor networks have been under investigation and it has been shown that mobility, while complicating the design of higher layer algorithms, also can improve the network, for instance, in terms of capacity, coverage, etc. [10]-[11]. Optimum mobility patterns for certain applications are proposed, such as mobile target tracking, chemical detection, etc. using both ground and aerial vehicles. Mobile robots with swarming capability operate cooperatively and aim to achieve a global goal have also been considered [12]-[16]. 


\subsection{Mobility Models}

There are several mobility models that take into account the dependence on the mobility pattern of other nodes in the network [4], social relationships of the mobile nodes [5], or topographical information [6], etc. In this paper, the following mobility models are considered:

- Random Walk: A mobile node picks a random speed and direction from pre-defined uniform distributions either at fixed time intervals or after a certain fixed distance is traveled. The current speed and direction of the mobile node do not depend on the previous speeds and directions.

- Random Direction: A random direction drawn from a uniform distribution is assigned to a mobile node and the mobile node travels in that direction till it reaches the boundary of the simulation area. Once it reaches the boundary, it pauses there for a fixed amount of time, then moves along the new randomly selected direction. In this paper, for fair comparison, we assume that the pause time is zero.

- Parallel-formation: Mobile nodes sweep the geographical area from border to border following a direction parallel to the boundary line.

\subsection{Coverage-Based Mobility}

In this newly proposed mobility model, the objective is maximizing coverage in a given time duration. To this end, we aim to minimize the overlap between the coverage areas of different mobile nodes and as shown in Fig. 1, we model forces between mobile nodes that cause them to repel each other. The magnitude of the force that each node applies to others is inversely proportional to the distance between the nodes, i.e., the closer the nodes get the stronger they push each other. We also assume that the mobile node knows its current direction and a force with a magnitude inversely proportional to the node's transmission range (i.e., $r$ ) is applied to it in the direction of movement to avoid retracing the already covered areas by the mobile node. At the time of direction change, each mobile node computes the resultant force vector acting on them by themselves and their neighbors (i.e., the mobile nodes within their transmission range) and move in the direction of the resultant vector. The forces on mobile node 1 at the time of decision are illustrated in Fig. 1, where mobile node 1 is moving toward right in the previous step.

Observe from Fig. 1 1 that the resultant force on node $i, \boldsymbol{R}_{i}=\sum_{j} \boldsymbol{F}_{j i}$, where $\boldsymbol{F}_{i i} \|$ $\boldsymbol{V}_{i}$ with $\left|\boldsymbol{F}_{i i}\right|=\frac{1}{r}$ and $\left|\boldsymbol{F}_{j i}\right|=\frac{1}{d_{j i}}$ when $j \neq i$, where $\boldsymbol{V}_{i}$ is the velocity vector of mobile node $i, r$ is the transmission range of each mobile node, and $d_{i j}$ is the distance between nodes $i$ and $j$. The direction of $\boldsymbol{F}_{j i}$ is parallel to the line drawn from node $j$ to node $i$. Mobile node $i$ will move in the direction of $\boldsymbol{R}_{i}$ with a speed chosen from the range $\left[0, V_{m}\right]$ for a fixed time duration (i.e., a step length). Same algorithm is run for all the mobile nodes and the directions are updated accordingly. If, at the time of direction change, a mobile mode does not have any neighbors, the direction is not changed. Note that the step length is a design parameter and depends on the system parameters such as $N_{m}$ and $r$ among others.

Since the mobile airborne network is highly dynamic and the neighborhood of the mobile nodes constantly change, the mobile nodes need to decide based on only local 


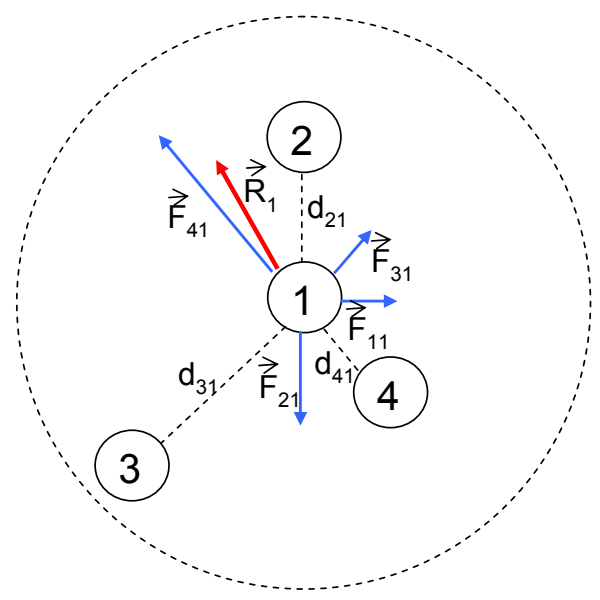

Fig. 1. Illustration of forces on mobile node 1, where the dashed circle is the transmission range of the node and mobile node 1 is moving toward right

interaction with other nodes and adapt to the changes in the environment in a distributed, self-organizing manner. Note that while the ultimate goal of maximizing coverage is not incorporated in the mobility model, as our results show a better coverage (i.e., event detection capability) emerges. Further work is necessary to design an analytical model that studies the performance of this mobility model and possibly to provide improvements.

\section{Event Detection Analysis of Parallel-Formation}

The system under investigation is a wireless sensor network that consists of airborne mobile nodes with the same transmission range. We assume that the UAVs fly at the same altitude and the directions are considered in a two-dimensional plane. The system parameters are summarized in Table 1 .

Table 1. System Parameters

\begin{tabular}{|l|l|}
\hline Parameter & Definition \\
\hline$N_{m}$ & Number of mobile nodes \\
\hline$r$ & Transmission range \\
\hline$a$ & Square simulation area length \\
\hline$P_{d}$ & Total event (target) detection probability \\
\hline$P_{m}$ & Event (target) miss probability for each UAV \\
\hline$t_{d}$ & Event (target) duration \\
\hline
\end{tabular}

In this section, we provide the event coverage (detection) probability by the mobile nodes flying in parallel formation (See Fig. 2 for a simple illustration) within a given time duration $t_{d}$. Since complete coordination between the mobile nodes is required, the mobile network needs to be connected at all times. 

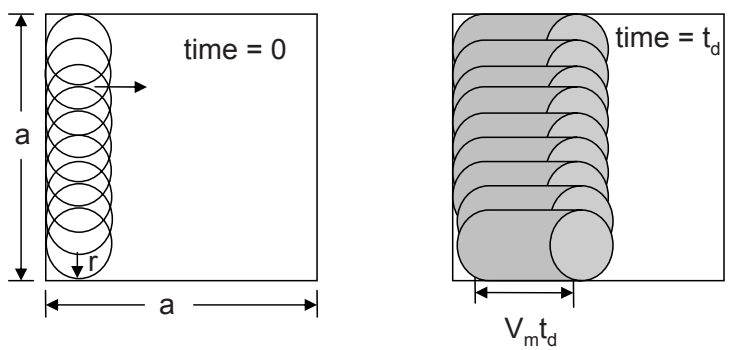

Fig. 2. Coverage illustration of mobile sensor nodes during time duration $t_{d}$, when the speed of the mobile nodes is $V_{m}$ for parallel-formation

Note that the event detection probability can be determined from the percentage area that is covered over time $t_{d}$. Assume that the transmission range of the mobile nodes is $r$ and their coverage area is of disc shape, i.e., area covered by each node at a given time is $\pi r^{2}$ and the nodes fly with a constant speed $V_{m}$. To better evaluate the limitations of the parallel-formation mobility, we assume that the number of mobile nodes, $N_{m}$ is such that one side of the square coverage area is fully-covered when the mobile nodes are aligned. Depending on the application of interest some overlap between the coverages of the UAVs might be assumed. In this work, we assume that the UAVs are placed such that the overlap is minimized given that airborne network is connected; i.e., the displacement between the UAVs is equal to $r$.

First, let's assume that the UAV sensors are accurate (i.e., probability of miss $\left(P_{m}\right)$ $=0$ ). The total covered area by the UAVs in time $t_{d}$, where $t_{d} \leq \frac{a}{V_{m}}$ is the sum of areas of the overlapping truncated cylinders shown in Fig. 2 and is given by:

$$
A_{c}=a V_{m} t_{d}+A_{o c}
$$

where $A_{o c}$ is the overlapping coverage between the discs and is given by:

$$
A_{o c}=\left\{\begin{array}{c}
\frac{2 a r-(4-\pi) r^{2}}{2}-\left(\frac{a-2 r}{r}\right) \frac{12 r^{2}-2 \pi r^{2}-3 \sqrt{3} r^{2}}{12} \\
0 \leq t_{d} \leq \frac{a-r}{V_{m}} \\
a(r-x)-2 A_{1}-\left(\frac{a-2 r}{r}\right) A_{2}, \\
\frac{a-r}{V_{m}} \leq t_{d} \leq \frac{a-r+0.5 r \tan (\pi / 12)}{V_{m}} \\
a(r-x)-2 A_{1}, \frac{a-r+0.5 r \tan (\pi / 12)}{V_{m}} \leq t_{d} \leq \frac{a}{V_{m}}
\end{array}\right.
$$

where

$$
\begin{aligned}
A_{1} & =(r-x)\left(r-\sqrt{r^{2}-(r-x)^{2}} / 2\right) \\
& -0.5 r^{2}\left(\frac{\pi}{2}-\arccos \left(\frac{r-x}{r}\right)\right) \\
A_{2} & =(r-x)\left(r-\sqrt{r^{2}-(r-x)^{2}}\right)-0.25 r^{2} \sqrt{3} \\
& -r^{2}\left(\frac{\pi}{6}-\arccos \left(\frac{r-x}{r}\right)\right) \\
x & =V_{m} t_{d}-(a-r)
\end{aligned}
$$


Then, the probability of detection $\left(P_{d}\right)$ for a single event, when $P_{m}=0$ is given by:

$$
P_{d}=\left\{\begin{array}{l}
\frac{A_{c}}{a^{2}}, 0 \leq t_{d} \leq \frac{a}{V_{m}} \\
1, \quad t_{d} \geq \frac{a}{V_{m}}
\end{array}\right.
$$

The probability of detection for a known number of $N_{t a r}$ targeted events, $P_{d_{m}}$, can be calculated by substituting $P_{d}$ from Eq. (6) into the following:

$$
P_{d_{m}}=\left(P_{d}\right)^{N_{t a r}}= \begin{cases}\left(\frac{A_{c}}{a^{2}}\right)^{N_{t a r}}, & 0 \leq t_{d} \leq \frac{a}{V_{m}} \\ 1, & t_{d} \geq \frac{a}{V_{m}}\end{cases}
$$

If on the other hand $P_{m} \neq 0$, i.e., there is a non-zero probability that an event may not be detected even if the whole area of interest is covered by the UAVs, then $P_{d}$ at a given time $t_{d}$ can be calculated to be:

$$
\begin{aligned}
P_{d} & =\left(1-P_{m}^{N_{c}}\right) \sum_{i=1}^{n-1} P_{m}^{N_{c}(n-1-i)} \\
& +P_{m}^{N_{c}(n-1)}\left(1-P_{m}^{N_{c}}\right) A_{c} / a^{2}
\end{aligned}
$$

where $n=\left\lceil\frac{V_{m} t_{d}}{a}\right\rceil$ is the total number of passes within $t_{d}, N_{c}$ is the number of checks a UAV does to detect an event, $A_{c}$ is the covered area within time $t_{d}-(n-1) a / V_{m}$ (i.e., the covered area during the n-th pass) and can be calculated using Eq. (1). Note that $N_{c}$ is a design parameter and depends on how often sensing is desired. As $N_{c}$ is increased, the effect of miss probability will be reduced, as expected.

\section{Results and Discussion}

In this section, performance comparison of several mobility models in terms of event detection probability is provided via Monte Carlo simulations, where each data point is computed over 2000 different runs. It is assumed that the range of the mobile nodes, $r$, is $500 \mathrm{~m}$. The simulation area is square-shaped with a length of $4000 \mathrm{~m}$. For parallelformation, these values correspond to 8 mobile nodes and the mobile nodes are initially aligned along one side of the observation area. For the other mobility models under study in this paper, initially, mobile nodes are randomly distributed in the simulation area. When a mobile node approaches the boundary of the simulation area, a random direction toward the simulation area is assigned for random walk and coverage-based mobility models. The speed of the mobile nodes is assumed to be $5 \mathrm{~m} / \mathrm{s}$. The directions of the mobile nodes are updated every $50 \mathrm{~m}$. Similarly, the step size for sensing is also assumed to be $50 \mathrm{~m}$. We assume that a single event occurs at a random location within the simulation area and lasts for a duration of $t_{d}$ seconds.

\subsection{Probability of Detection with Perfect Sensing}

In this section, we study the probability of detection performance of several mobility models when the sensors on-board the UAVs are accurate with $P_{m}=0$. Fig. 3 shows the 


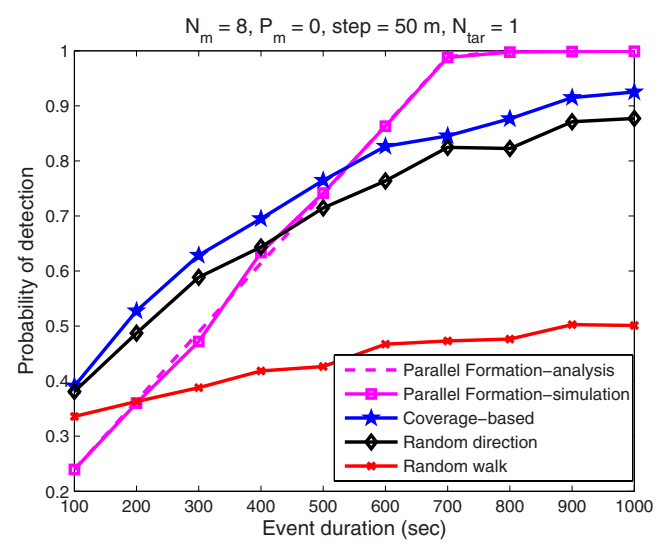

Fig. 3. Probability of detection versus event duration, when $N_{m}=8, P_{m}=0$, and number of targeted events is 1

probability of detection versus $t_{d}$ for random walk, random direction, coverage-based and parallel-formation models when $N_{m}=8$. Observe that the detection performance of the mobility models under investigation strictly depends on the timing constraints of the application. If $t_{d} \geq a / V_{m}=800 \mathrm{sec}$, parallel-formation outperforms the rest of the models since the whole geographic area can be swept by then. However, if the timing constraints are strict and do not allow a network flying in formation to cover the area, then a more efficient mobility model is required. The simple coverage-based mobility model that inherently reduces the overlapped coverage areas between different mobile nodes can perform better than the rest, only using local information. More sophisticated mobility models can be designed that take into account the history of flight of the UAVs; however, this is beyond the scope of this paper.

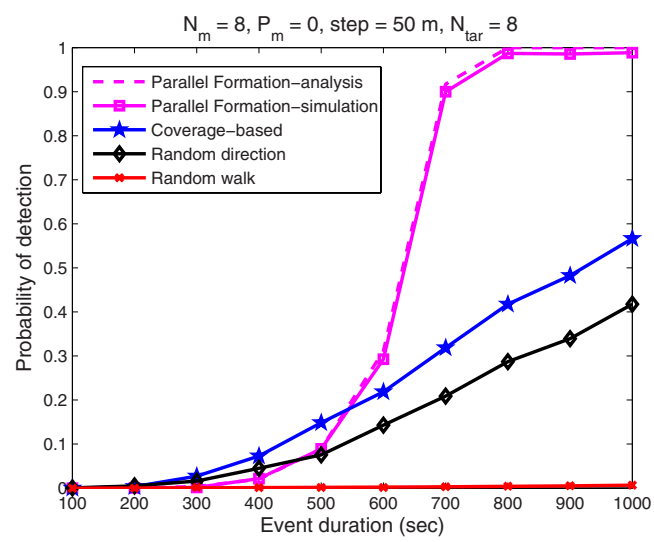

Fig. 4. Probability of detection versus event duration, when $N_{m}=8, P_{m}=0$, and number of targeted events is 8 
As an illustration, we also studied the case with multiple stationary events. Fig. 4 shows the probability of detection versus event duration when the number of targeted events is 8 . Note that while for short time values the probability of detection performance of all models suffer, the trends are still the same as the single event scenario. The random mobility models fail to detect all events as fast as parallel-formation. Also, observe from Figures 3 and 4 that the analytical and simulation results for parallelformation are in excellent agreement verifying the findings, where the analytical results are obtained using Eq.'s (6) and (7), respectively.

\subsection{Probability of Detection with Imperfect Sensing}

Next, we study the case with imperfect sensing capabilities. We investigate the impact of event duration as well as the probability of miss of the sensors on the detection

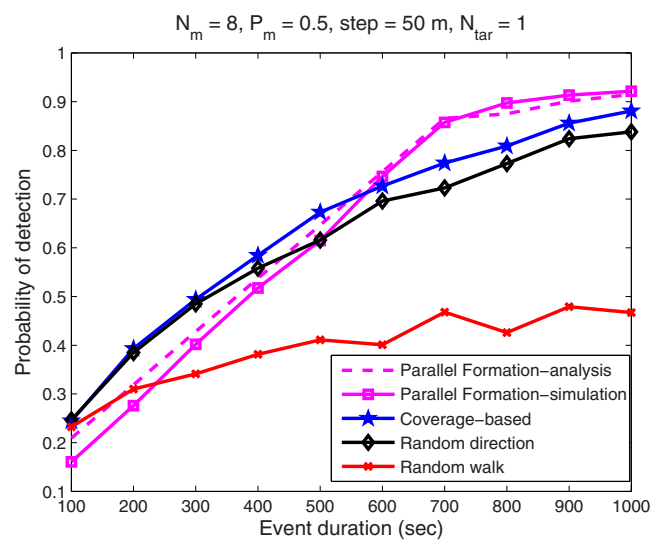

(a)

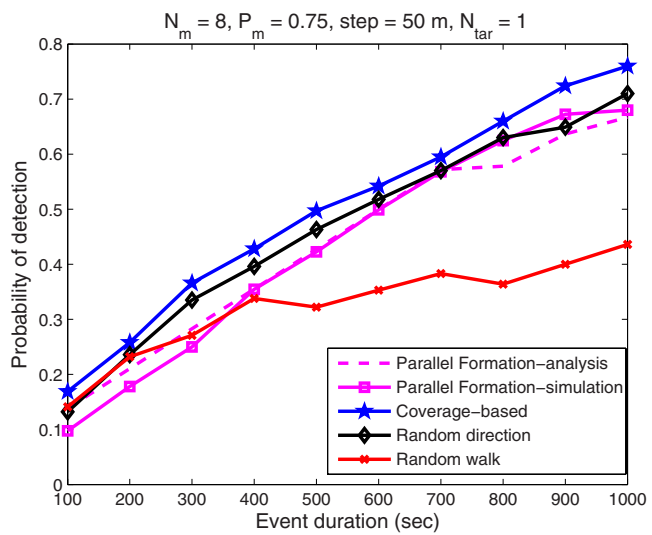

(b)

Fig. 5. Probability of detection versus event duration, when $N_{m}=8$ and (a) $P_{m}=0.5$ and (b) $P_{m}=0.75$ 
performance of the chosen deterministic and random mobility models. Simulation and analytical results are provided, where the analytical results are obtained using Eq. (8).

Fig. 5 shows the probability of detection versus event duration when $P_{m}=$ $\{0.5,0.75\}$. In both cases, the probability of detection decreases with respect to the case with $P_{m}=0$, as expected. Observe that parallel-formation significantly suffers from the imperfections of the sensing capabilities and although the whole observation area can be fully-swept, probability of detection stays at 0.9 and 0.6 for $P_{m}=0.5$ and $P_{m}=0.75$, respectively. While for $P_{m}=0.5$, parallel-formation can still perform better than the other models for certain time durations, when $P_{m}$ is increased 0.75 , coverage-based mobility model performs consistently better than parallel-formation.

To better illustrate the impact of $P_{m}$ on the performance, we studied the detection performance when $t_{d}=100,1000 \mathrm{sec}$ for several $P_{m}$ values. Results are shown in Fig.6 Observe that when $t_{d}=100 \mathrm{sec}$, all random models outperform parallel-formation,

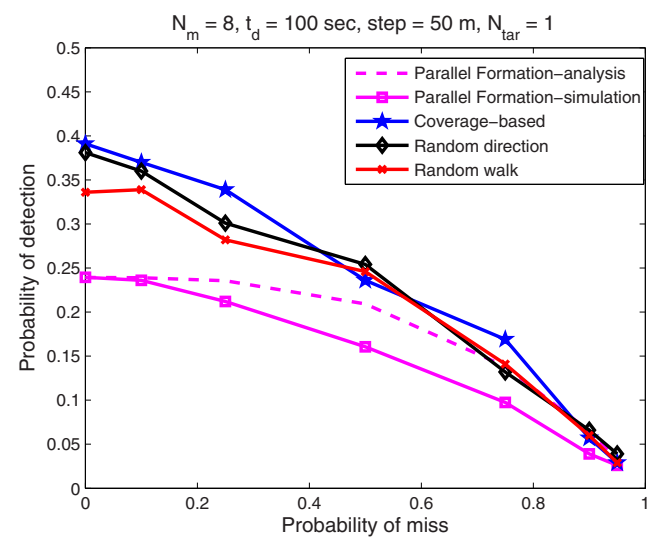

(a)

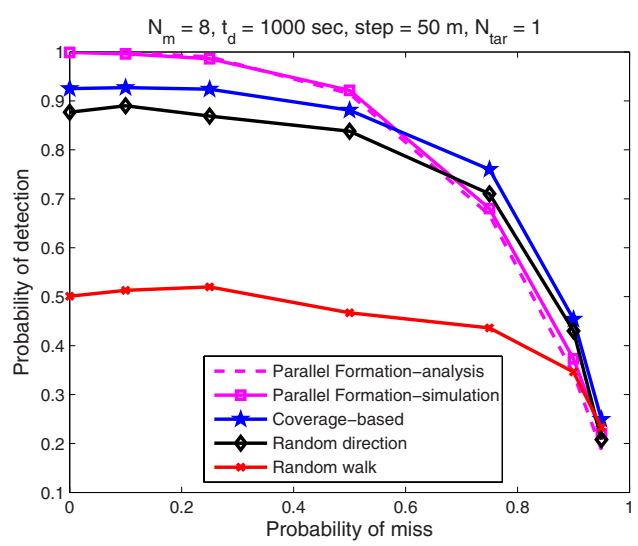

(b)

Fig. 6. Probability of detection versus probability of miss, when $N_{m}=8$ and (a) $t_{d}=100 \mathrm{sec}$ and (b) $t_{d}=1000 \mathrm{sec}$ 
for all $P_{m}$ values under investigation. On the other hand, when the timing constraints are loosened parallel-formation, coverage-based and random direction models all perform similarly. Random models exceed the performance of parallel-formation when the sensing capabilities are highly imperfect.

Finally, while not analyzed in this paper, a drawback of the centralized scheme is the requirement to be fully-connected at all times. In the configuration under study in this paper if a node in the middle of the formation breaks down the network itself becomes disconnected and a percentage of the area cannot be covered anymore, unless the remaining nodes regroup into a new formation. On the other hand, intuitively, the distributed mobility models studied in the paper are expected to be more robust to node failures, since system-wide connectivity is not required and the nodes communicate with each other only when they are within each other's coverage (for the coverage-based mobility) or do not communicate at all (random walk, random direction). The impact of malfunctioned nodes on the detection performance needs to be further investigated.

\section{Conclusions}

In this work, event detection performance of an airborne UAV sensor network that employs deterministic and random mobility models is investigated. Specifically, the limitations of a UAV network flying in parallel-formation is explored and its performance is compared with some legacy mobility models as well as a cooperative, coverage-based mobility model that uses local topology information. The results show that if timingconstraints are highly-stringent or the sensing capabilities on-board the UAVs are highly imperfect, parallel-formation might not be sufficient to detect the events in the observation area. While for such cases random mobility models are shown to improve the detection performance, further study is necessary to design an optimum mobility pattern that minimizes the event detection time and/or maximizes the probability of detection.

\section{Acknowledgements}

The author would like to thank Prof. Christian Bettstetter of University of Klagenfurt and the attendees of Lakeside Research Days 2009 for valuable discussions and feedback on the paper. This work was supported by Lakeside Labs GmbH, Klagenfurt, Austria and funding from the European Regional Development Fund and the Carinthian Economic Promotion Fund (KWF) under grant 20214/17095/24772.

\section{References}

1. Akyildiz, I.F., Su, W., Sankarasubramaniam, Y., Cayirci, E.: A Survey on Sensor Networks. IEEE Comm. Mag. 40(8), 102-114 (2002)

2. Sohrabi, K., Gao, J., Ailawadhi, V., Pottie, G.J.: Protocols for Self-organization of a Wireless sensor network. IEEE Pers. Comm. 7(5), 16-27 (2000)

3. Quaritsch, M., et al.: Collaborative Microdrones: Applications and Research Challenge. In: Proc. Intl. Conf. Autonomic Computing and Comm. Sys., September 2008, p. 7 (2008) 
4. Camp, T., Boleng, J., Davies, V.: A Survey of Mobility Models for Ad Hoc Network Research. Proc. Wireless Commun. and Mobile Comp (WCMC): Special issue on Mobile Ad Hoc Networking: Research, Trends and Applications 2(5), 483-502 (2002)

5. Musolesi, M., Mascolo, C.: A Community Based Mobility Model for Ad Hoc Network Research. In: Proc. ACM/SIGMOBILE Intl. Workshop on Multi-hop Ad Hoc Networks: from theory to reality (REALMAN 2006), May 2006, pp. 31-38 (2006)

6. Jardosh, A., Belding-Royer, E.M., Almeroth, K.C., Suri, S.: Towards Realistic Mobility Models for Mobile Ad hoc Networks. In: Proc. Intl. Conf. Mobile Computing and Networking (MobiCom 2003), September 2003, pp. 217-229 (2003)

7. Wang, X., Xing, G., Zhang, Y., Lu, C., Pless, R., Gill, C.: Integrated Coverage and Connectivity Configuration in Wireless Sensor Networks. In: Proc. Int's. Conf. Emb. Net. Sens. Sys (SenSys 2003), pp. 28-39 (2003)

8. Megerian, S., Koushanfar, F., Potkonjak, M., Srivastava, M.B.: Worst and Best-Case Coverage in Sensor Networks. IEEE Trans. Mob. Comp. 4(1), 84-92 (2005)

9. Liu, B., Towsley, D.: A Study of the Coverage of Large-scale Sensor Networks. In: Proc. IEEE Int'l. Conf. Mob. Ad hoc Sens. Sys (IEEE MASS 2004), October 2004, pp. 475-483 (2004)

10. Grossglauser, M., Tse, D.N.C.: Mobility Increases the Capacity of Ad Hoc Wireless Networks. IEEE/ACM Trans. Networking 10(4), 477-486 (2002)

11. Liu, B., Brass, P., Dousse, O., Nain, P., Towsley, D.: Mobility Improves Coverage of Sensor Networks. In: Proc. ACM Int'l. Symp. Mob. Ad hoc Net. Comp (ACM MobiHoc 2005), pp. 300-308 (2005)

12. Poduri, S., Sukhatme, G.S.: Constrained Coverage for Mobile Sensor Networks. In: IEEE Int'1. Conf. Robotics and Autom (IEEE ICRA 2004), April 2004, pp. 165-172 (2004)

13. Vincent, P., Rubin, I.: A Framework and Analysis for Cooperative Search Using UAV Swarms. In: Proc. ACM Symp. on Applied Computing (2004)

14. Jin, Y., Liao, Y., Minai, A.A., Polycarpou, M.: Balancing Search and Target Response in Cooperative Unmanned Aerial Vehicle (UAV) Teams. IEEE Trans. On Systems, Man, and Cybernetics 36(3) (2006)

15. Kovacina, M.A., Palmer, D., Yang, G., Vaidyanathan, R.: Multi-Agent Algorithms for Chemical Cloud Detection and Mapping Using Unmanned Air Vehicles. In: IEE/RSJ Intl. Conf. on Intelligent Robots and Systems (2002)

16. Palat, R.C., Annamalai, A., Reed, J.H.: Cooperative Relaying for Ad hoc Ground Networks Using Swarms. In: Proc. IEEE Milit. Comm. Conf (MILCOM 2005), October 2005, vol. 3, pp. 1588-1594 (2005) 